\title{
The Spreading of Information in Online Social Networks through Cellular Automata
}

\author{
Yuda Wang (i) and Gang Li \\ School of Economics and Management, Beijing University of Posts and Telecommunications, Beijing 100876, China \\ Correspondence should be addressed to Yuda Wang; wangyd@bupt.edu.cn
}

Received 10 September 2018; Accepted 15 October 2018; Published 1 November 2018

Academic Editor: Dimitri Volchenkov

Copyright (c) 2018 Yuda Wang and Gang Li. This is an open access article distributed under the Creative Commons Attribution License, which permits unrestricted use, distribution, and reproduction in any medium, provided the original work is properly cited.

\begin{abstract}
Epidemic dynamics in complex networks have been extensively studied. Due to the similarity between information and disease spreading, most studies on information dynamics use epidemic models and merely consider the characteristics of online social networks and individual's cognitive. In this paper, we propose an online social networks information spreading (OSIS) model combining epidemic models and individual's cognitive psychology. Then we design a cellular automata (CA) method to provide a computational method for OSIS. Finally, we use OSIS and CA to simulate the spreading and evolution of information in online social networks. The experimental results indicate that OSIS is effective. Firstly, individual's cognition affects online information spreading. When infection rate is low, it prevents the spreading, whereas when infection rate is sufficiently high, it promotes transmission. Secondly, the explosion of online social network scale and the convenience of we-media greatly increase the ability of information dissemination. Lastly, the demise of information is affected by both time and heat decay rather than probability. We believe that these findings are in the right direction for perceiving information spreading in online social networks and useful for public management policymakers seeking to design efficient programs.
\end{abstract}

\section{Introduction}

Networks such as Internet, communication networks, and social networks can be used to describe the interconnections among individuals. Since most of these networks have nontrivial structural properties, they are called complex networks [1-4]. According to the degree of each network node, complex networks are divided into regular networks, Erdos-Renyi (ER) networks [5], small-world networks [6], and scale-free (SF) networks [7]. Among those networks, the small-world model proposed by Watts and Strogatz describes the features of high clustering and small average path length, which is most suitable for real social networks. These small-world features [8] have been found to have a significant impact on the dynamics in networks [9].

Spreading [10] is one of the dynamical processes on complex networks, especially the spreading of epidemic, which has been investigated for decades and achieves a lot of theories. Most of these studies investigate susceptible-infectedsusceptible (SIS), susceptible-infected-recovered (SIR), or susceptible-exposed-infected-removed (SEIR) model [11-15]. With the growth of the Internet scale and the convenience of we-media, information such as rumors' spreading and evolution in online social networks has received special attention $[16,17]$. Due to the similarity between information and disease, the models mentioned above are widely considered.

Qian [18] investigated the spreading and evolution of public opinion on Weibo [19] with SIR model. Xiong et al. [20] proposed a new model called SCIR to describe the spreading of information. Centola et al. [21] have illustrated the effects of network structure on diffusion experimentally by studying the spread of health behavior through artificially structured online communities. However, all these studies are based on a probabilistic approach, regardless of individual's cognitive psychology and the characteristics of online social networks. Recently, researchers start to seriously take into consideration the specific features of online social networks information spreading, such as individual's characteristics and the impact of friends around [22-31]. Nevertheless, there are more factors affecting online information spreading. 
Furthermore, most previous studies of dynamical processes on complex networks are based on mean field method [32] or analytical equation [33]. However, these approaches has some serious drawbacks. The method based on mean field can only reflect an approximate trend from macroscopic view, which cannot accurately describe the spreading state of each individual. Meanwhile, analytical equation neglects variable susceptibility of individuals and cannot handle the different boundary and initial condition; in addition, it will be very complicated for large-scale online social networks. Cellular automata (CA) $[34,35]$ proposed by Von Neumann can overcome the above drawbacks and have been used by several researchers as an alternative method of modeling epidemics. In this model every cell of the grid represents an individual, updating state of each cell by state transition criterion $[36,37]$.

In summary, complex networks provide theoretical basis for social network topology, and the extensive investigation of epidemic models promotes the quantitative study of information spreading. By referring to complex network dynamics, as well as combining the features of public opinion, a breakthrough has been made in the research on information transmission in social networks. With the rapid development of mobile Internet and Internet of things, the online social network is fundamentally different from the traditional social network. The spreading and control of information, especially public opinion, has become a difficulty and key point concerned by public management policymakers. Meanwhile, due to the complexity of network topology and the explosion of network access nodes, traditional analytical methods have been unable to accurately calculate the results. By introducing the cellular automata theory, the bottleneck can be solved through the iterative calculation.

In this paper, we propose an online social networks information spreading (OSIS) model combining individual's cognitive psychology with traditional SEIR model. Moreover, we provide a computational method to prove the accuracy and authenticity of OSIS by cellular automata. We obtained experimental results with the help of computer graph theory. The results reveal that individual's cognition affects online information spreading. When infection rate is low, cognitive psychology prevents the spreading. By contrast, when infection rate is sufficiently high, it promotes transmission. We also demonstrate that the explosion of online social network scale and the convenience of we-media can greatly increase the ability of information dissemination, especially rumors. Finally, we show that the demise of information is affected by the dual effects of the decay of time and heat. In particular, the major contributions of our work can be summarized as follows:

(i) a new information spreading model that takes into account individual's cognitive psychology and the characteristics of online social networks: herd mentality factor, memory superposition factor, and attention attenuation factor;

(ii) a two-dimensional CA method that can accurately analyze the process of online information spreading; (iii) revealing the characteristic of online social networks information dissemination.

The sections in this paper are structured as follows: Section 1 presents the background while Section 2 describes the model; Section 3 shows the design of cellular automata followed by the experimental results in Section 4; finally, we conclude our paper in Section 5 with the future work discussed.

\section{Online Social Network Information Spreading Model}

2.1. Analysis of Information in Online Social Network. Compared with traditional social networks, online social networks use Internet as the medium of communication rather than face-to-face, which makes information transmission more easy to forward. Meanwhile, in social networks such as Twitter, information is transmitted synchronously to all friends, which is different from the peer-to-peer communication of offline networks. The communication of information is more highly timely. Meanwhile, because of the intelligence of terminals, the carrier of online information varies from text to sound, picture, video, etc. The content becomes interesting and easy to understand. Based on the features described above, we consider the information spreading model from three aspects.

(i) Propagation factor: Due to the characteristics of online social networks and individual's fast-food reading habits, the cognition of information depends not only on the content itself, but also on the heat. The more the friends who are concerned around a topic, the higher the probability that an individual will spread it.

(ii) Memory superposition factor: Online social networks shorten the distance among people, which makes people access the same information over a continuous period of time; we call this phenomenon memory superposition. The memory superposition enhances people's curiosity, thus prompting the spreading.

(iii) Information attenuation factor: In traditional epidemic models, a disease is cured via a certain probability, whereas the demise of information is affected by both time and heat decay.

2.2. Description of OSIS Model. Since information spreading is similar to epidemic, we propose our online social network information spreading (OSIS) model by combining the characteristics of online social networks mentioned above with epidemic SEIR model. In OSIS, each individual adopts one of four states: unaware, concerned, interested, and abandoned. Unaware represents the individual who has not heard the information and has the potential to accept it. Concerned stands for the person who is aware of the news but not willing to transmit it currently. Interested means the one believes in the news and has the ability to spread it. And abandoned denotes the individual who loses interests and never transmits the information again. We use complex 


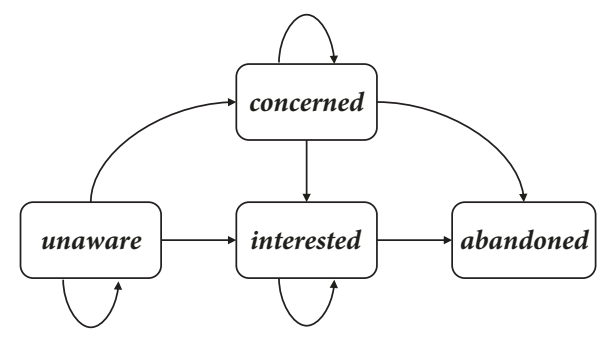

Figure 1: The state transfer diagram.

networks as the model of real social networks, in which each node and edge present individual and online social relationship.

As shown in Figure 1, the state of each node can be transformed over time. Initially, we randomly pick up one node as an interested node and the rest are in unaware state. At each time step, an unaware state individual receives a topic from her neighbor and turns to interested or concerned state according to the propagation factor. Furthermore, whether or not a concerned state node turns to interested state is based on memory superposition factor. Additionally, information attenuation factor determines interested to abandoned state.

2.3. The Algorithm. Based on OSIS model described above, an algorithm for simulation of information spreading on complex networks has been developed. The core idea in Algorithm 1 is presented with pseudocode.

(a) If a node is in unaware state and there are interested state nodes around, propagation factor determines state transition. In our model, the dissemination of information is due to not only the information itself, but also to herd mentality. For that reason, the propagation factor consists of two parts. One part is due to the information itself, here we adopt the algorithm in traditional SIR model. The infection rate is $\alpha$ and the number of interested neighbors is $\mathrm{m}(\mathrm{t})$ at the th time. The node will have a probability of $\mathbf{1}-(\mathbf{1}-\boldsymbol{\alpha})^{\boldsymbol{m}(\boldsymbol{t})}$ being infected. The other part is caused by herd mentality, which includes both positive and negative effect. We assume that for a given individual at the th time, she has $\mathrm{k}$ adjacent, $\mathrm{m}(\mathrm{t})$ interested, and $n(t)$ abandoned neighbors. The proportion of interested individuals in the neighbors determines the positive influence of the herd effect. When no one around propagates the information (i.e., $\boldsymbol{m}(\boldsymbol{t}) / \boldsymbol{k}$ $=0$ ), the influence probability of herd effect is 0 . While all friends are in interested state (i.e., $\boldsymbol{m}(\boldsymbol{t}) / \boldsymbol{k}$ $=0$ ), the influence of herd effect is the greatest, the probability of which is 1 . Our observations show that the effect of this ratio is a positively exponential effect rather than a linear effect; we define the effect as $\left(1-e^{-m(t) / k}\right) /(1-1 / e)$. By contrast, if most friends are in abandoned state, this may reduce the transmissibility. The more the neighbors in abandoned state, the lower the probability that the truth of the information

\section{Define:}

(1) State $\longleftarrow$ current state of a node

(2) $\tau \longleftarrow$ time step

(3) Duration $\longleftarrow$ the period in current state

Procedure:

(4) for each $t \in \tau$ do

(5) Traverse the network and fill in State of each node

(6) for each node in small-world networks do

(7) if State = unaware then

(8) if Propagation factor then

(9) State $\longleftarrow$ interested

(10) else

(11) State $\longleftarrow$ concerned

(12) end if

(13) else if State $=$ concerned then

(14) if Memory superposition factor then

(15) State $\longleftarrow$ interested

(16) else

(19)

(20)

(21)

(22)

(23)

(24)

(26)

(27) end for

$$
\text { else }
$$

end if

else if State $=$ interested then

if Information attenuation factor then State $\longleftarrow$ abandoned

else

Duration $\longleftarrow$ Duration +1

end if

end if

end for

Algorithm 1: The information spreading model pseudocode.

is believed, which reduces the probability of spreading. Similarly, we use the proportion of abandoned individuals in the neighbors to calculate the impact of herd effect, and the expression is $\left(\mathbf{1}-\boldsymbol{e}^{-\boldsymbol{n}(\boldsymbol{t}) / \boldsymbol{k}}\right) /(\mathbf{1}-\mathbf{1} / \boldsymbol{e})$. Above all, the herd mentality effect can be described as $\left(\left(1-e^{-m(t) / k}\right)-\left(1-e^{-n(t) / k}\right)\right) /(1-1 / e)$.

In this paper the propagation factor is composed of these two parts by the assigned $\mathbf{1 / 2}$ weight for the expressiveness of the model and the computability of simulations, which can be expressed in (1). The greater $p(t)$, the more possibility of transferring state from unaware to interested.

$$
\begin{aligned}
p(t)= & \frac{1}{2} *\left(1-(1-\alpha)^{m(t)}\right)+\frac{1}{2} \\
& * \frac{\left(1-e^{-m(t) / k}\right)+\left(e^{-n(t) / k}-1\right)}{1-1 / e}
\end{aligned}
$$

(b) If a node is in concerned state, memory superposition factor determines state transition. In our designed model, we believe that human memory has an impact on the spread of information. It is easy for an individual in concerned state to change their state by constantly having neighbors to spread or reject the message within a short period of time. When 
different friends forward the same topic multiple times, it will promote the concerned state to become the interested state. By contrast, if not heard for a long time, the topic will be abandoned. We define the one who receives the information over the time as $s(t)$, which quantitatively describes the cumulative effect of the memory. Initially set $s(t)$ to 0 ; i.e., the variable is 0 when a node changes from an unaware state to a concerned state for the first time at th time step. If there are new turned interested nodes around the next time step, then $s(t+1)=s(t)+1$. Otherwise, $\mathrm{s}(\mathrm{t}+1)=\mathrm{s}(\mathrm{t})-1$. We set threshold $\varepsilon$. When memory superposition factor accumulates above $\varepsilon$, the state turns to interested. Contrarily if $s(t)<-\varepsilon$, it becomes abandoned state.

(c) If the node is in interested state, information attenuation determines state transition. Attenuation is a compound process; here we consider both the interest decay and time decay. We use exponential function to represent time decay and the proportion of interested individuals in the neighbors to represent interest decay. Thus information attenuation factor can be expressed as (2). We set threshold $\delta$; when a(t) decays below the threshold, the interested state node will turn to abandoned state.

$$
a(t)=e^{-t} * \frac{m(t)}{k}
$$

\section{Design of Cellular Automata}

Cellular automata (CA) constitute the model of physical systems, where space and time are discrete and interactions are local. It is an effective theoretical tool for studying complex systems and, at the same time, can be simulated exactly by computer program because of their intrinsic discreteness. For those reasons, we adopt CA to realize OSIS model. In CA the value of each cell presents the local state, which can only interact with its neighbors and is incapable of global communication. The state is updated simultaneously at discrete time steps according to the states of their neighbors at the preceding time step. Here are the elements of our proposed cellular automata.

(a) Cellular space: Our model is set up in complex network; each node in the network consists of the cellular space.

(b) Cellular neighborhood: For the reason that our cellular space is not the regular two-dimensional network, we cannot adopt traditional Von Neumann or Moore structure. The neighborhood in Figure 2 illustrates that the nodes associated with edges can be regarded as adjacent relationship, which we call neighbors.

(c) Cellular state: According to OSIS model, the state of each cell is unaware, concerned, interested, or abandoned.

(d) State transfer rule: As the methodology described above, unaware state turns to whether interested or concerned state is decided by propagation factor.

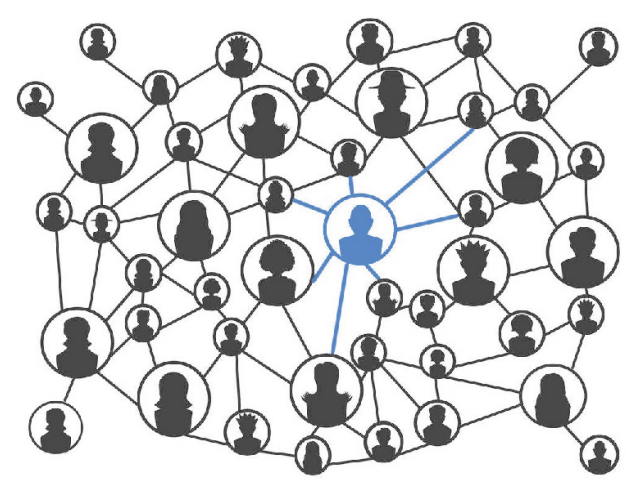

FIgURE 2: The neighborhood of each cell is formed by the cells associated with edges.

\begin{abstract}
Memory superposition factor is able to determine concerned node's next state. Furthermore, information attenuation factor can turn interested to abandoned state. The detailed transfer relationship can be found in Figure 1.
\end{abstract}

\section{Experiments and Evaluation}

4.1. Implementation of Online Social Network. We use smallworld network to simulate online social network in our experiments. On the one hand, small-world theory is also called six degrees of separation, which means the distance between two strangers is at most six people. This feature is the closest to the online social network. On the other hand, on the basis of sampling and analysis on millions of level data excavated from the social groups in [38], online social network has small-world effect.

The implementation of a small-world network comes from a regular lattice with a linear size $\mathrm{L}$ and with $\mathrm{N}=$ $\mathrm{L} * \mathrm{~L}$ nodes. The initial node degree is $\mathrm{k}$, which means each node is connected to its $\mathrm{k} / 2$ nearest neighbors clockwise and counterclockwise, deleting one link randomly, adding a link between two randomly chosen nodes with probability $\mathrm{p}$, and avoiding duplicate links and self-loops. The link probability $p$ turns the nature of the network between that of a regular network $(\mathrm{p}=0)$ and that of a random network $(\mathrm{p}=1)$.

4.2. Experimental Results. In our experiments, we use $\mathrm{C}++$ programming language to build the small-world networks with 2500 nodes and an average degree of 6 . The shortest distance between two nodes is calculated by Dijkstra's algorithm in computer graph theory. The results are averaged over 100 realizations.

4.2.1. The Comparison with Traditional SEIR Model. To demonstrate the effectiveness of OSIS, we compare our model with traditional epidemics SEIR model. We firstly observe the information spreading over time and space, considering various infection rates. Afterwards, we illustrate the evolution. Particularly, unaware, concerned, interested, and abandoned state correspond to susceptible, exposed, infected, and recovered, respectively, in SEIR. 


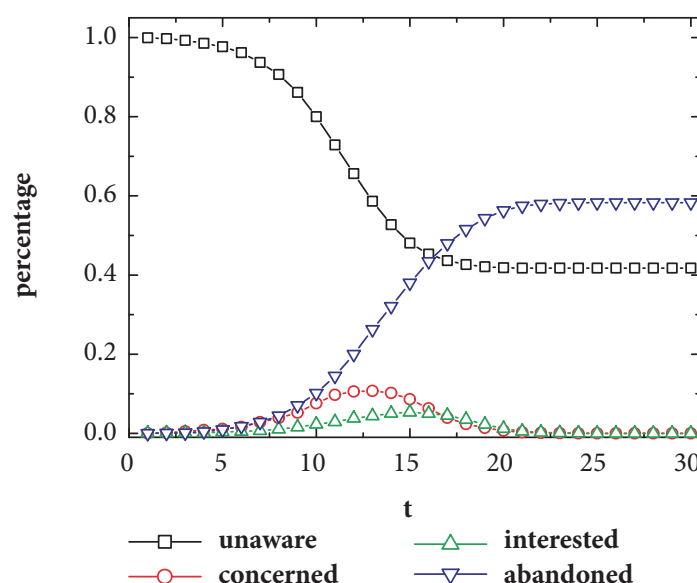

(a)

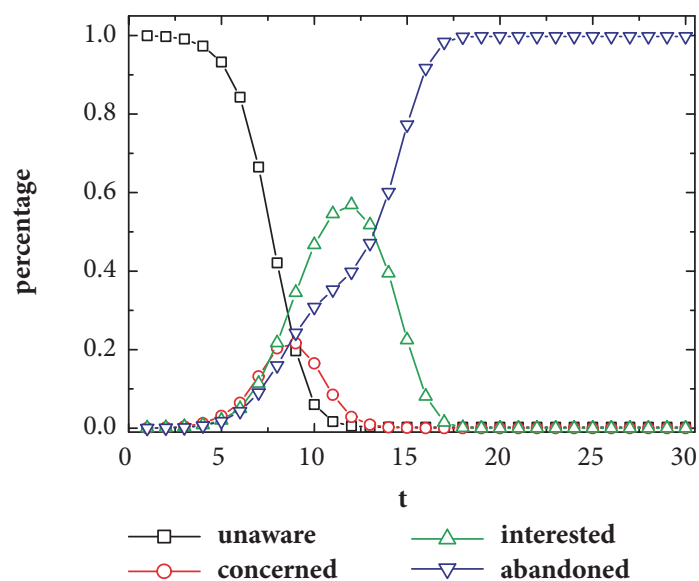

(c)

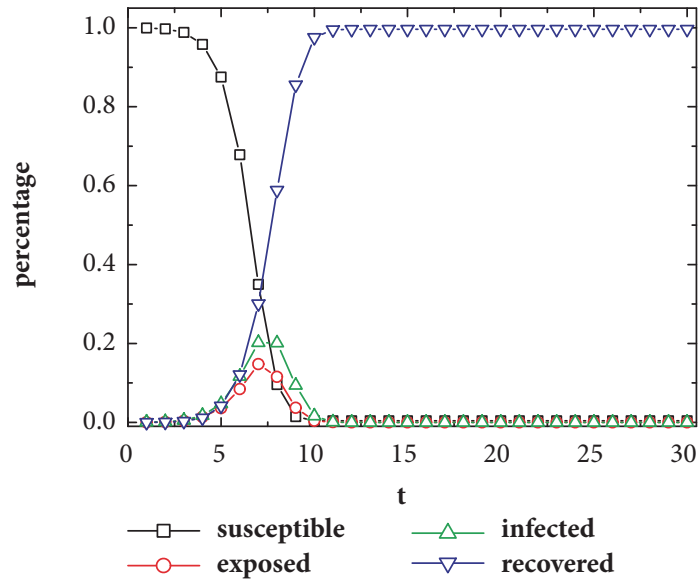

(e)

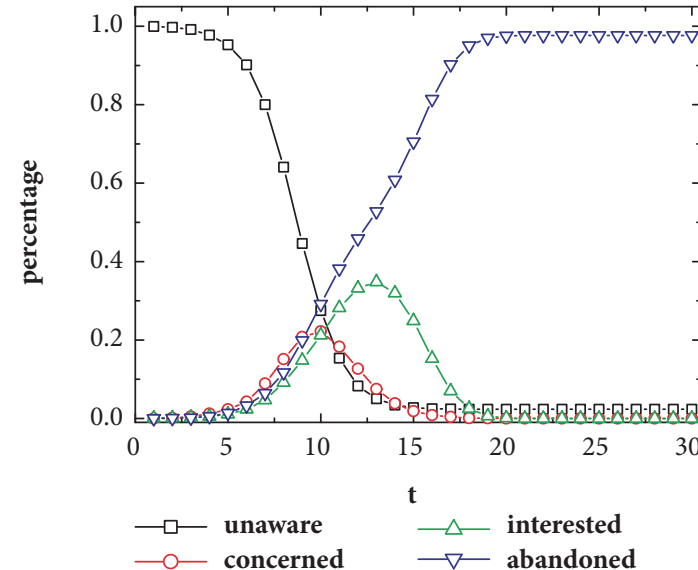

(b)

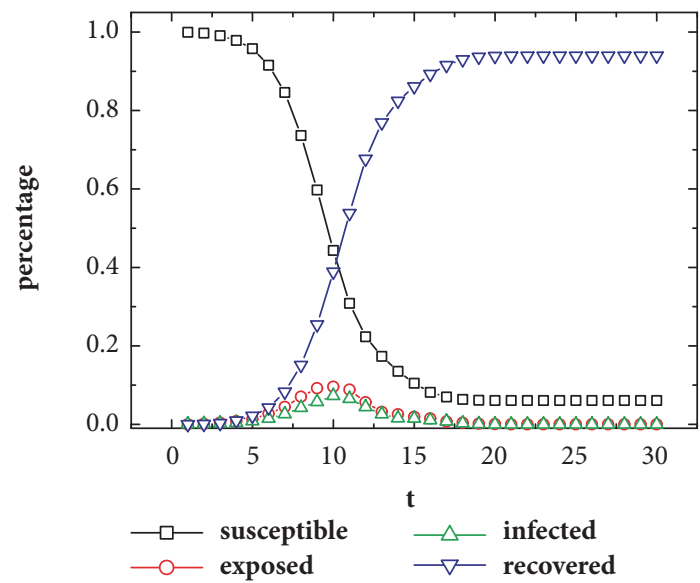

(d)

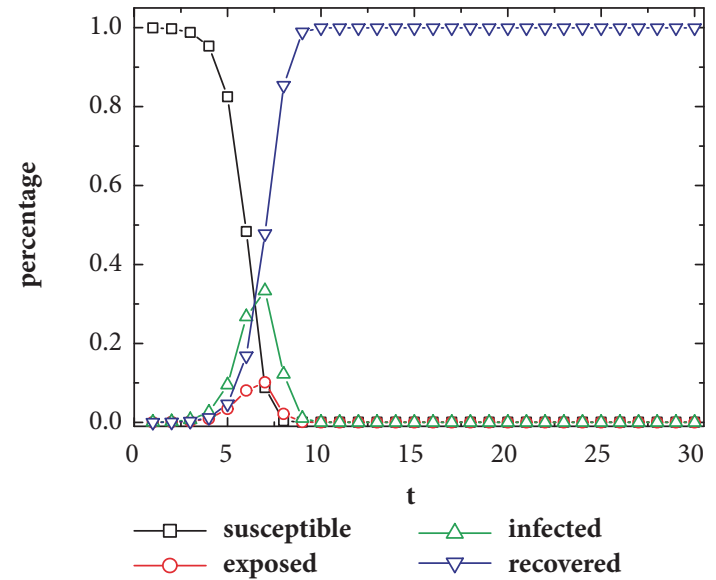

(f)

FIgURE 3: The percentage of each state in a continuous period of time. The spreading model is OSIS ((a), (b), (c)) and SEIR ((d), (e), (f)) separately. The infection rate is 0.35 in (a) and (d), 0.55 in (b) and (f), 0.75 in (c) and (e).

Firstly, we set up three sets of contrastive experiments. Without generality, we set the rewiring probability of the small-world networks 1, i.e., a "complete" small-world network. And the threshold of the memory superposition factor is 1 and the threshold of information attenuation is 0.5 . The infection rate is 0.35 and 0.55 in the first and second set, which is 0.75 in the third one.

Initially, one node in the centre is infected. After 30 time periods, the percentage of each state is recorded and the result is shown in Figure 3. 


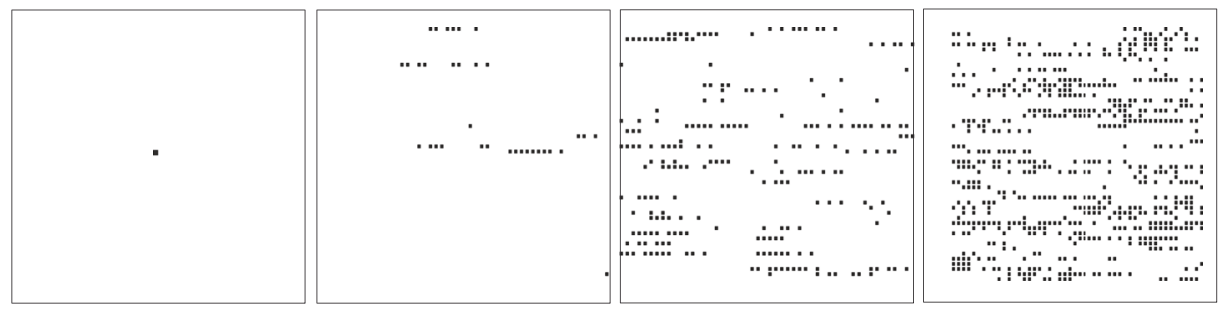

(a)

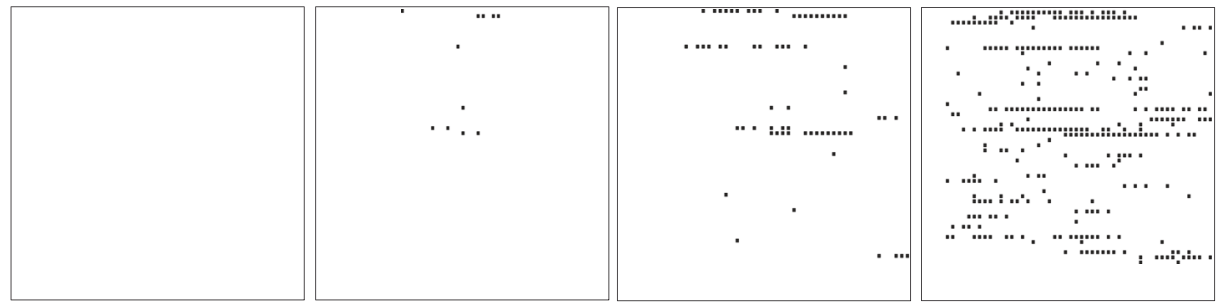

(b)

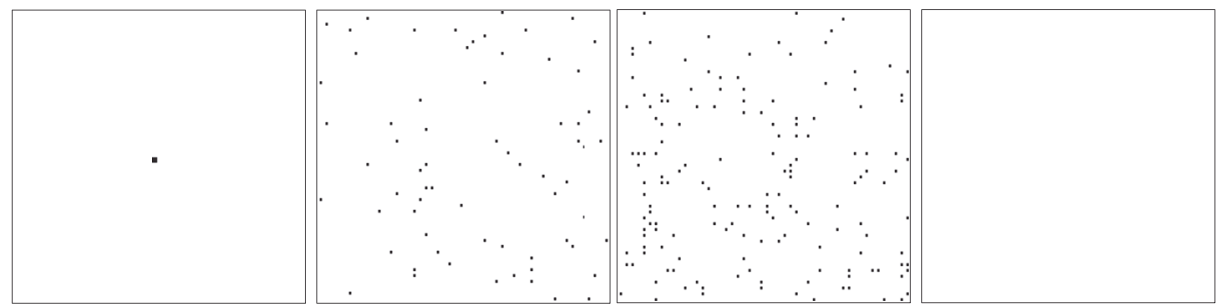

(c)

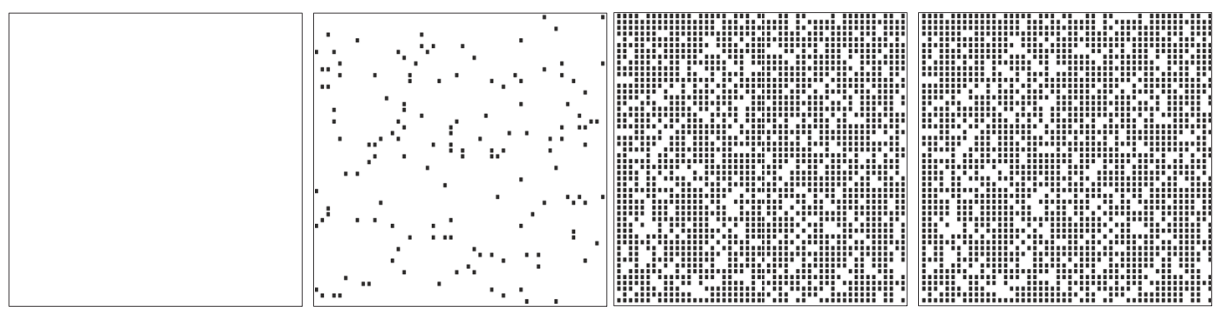

(d)

t

FIgURE 4: The information spreading evolution in small-world. The spreading model is OSIS ((a), (b)) and SEIR ((c), (d)) separately. (a) and (c) present the evolution of interested nodes, (b) and (d) stand for abandoned nodes. The infection rate is 0.35 . The time period is $0,5,10,15$.

It can be seen from Figures 3(a) and 3(d) that when infection rate is low, the percentage of nodes in final abandoned state is $94 \%$ in SEIR, which decreases as much as $38 \%$ to $58 \%$ in OSIS. This is because when the number of initial infected nodes as well as information spreading capacity is low, herd mentality inhibits the transmission of information. The phenomenon explains herd mentality effect on online social networks from the reverse. Figures 3(b) and 3 (e) illustrate the peak of interested nodes is significantly delayed, which is from the 7th time step in SEIR to the 13th time step in OSIS. The result reveals that both propagation and disappearance of a topic is gradual over time or with the attention of people around, which is different from epidemic. Figures 3(c) and 3(f) demonstrate that when the infection rate is high, the number of infected nodes is increased by $72 \%$ to 0.57 from 0.33 . This is due to the fact that herd mentality and memory superposition effect promote the spreading of information.

Furthermore, to discover the evolution of OSIS, we investigate the information spreading over space. In this experiment, rewiring probability of the small-world network is that 0.1 and 1 nodes in the centre of the network are infected initially. It can be seen from Figure 4 that the evolution of online social network information is a gradual process, while the disease transmission has the characteristics of sudden outbreak and the one exposed to the disease eventually develops antibodies. Further comparing Figures 4(a) and $4(\mathrm{c})$, it is evident that the disease with SEIR model spreads very fast and suddenly dies out. By contrast, the information spreads throughout the network smoothly. Meanwhile, the 


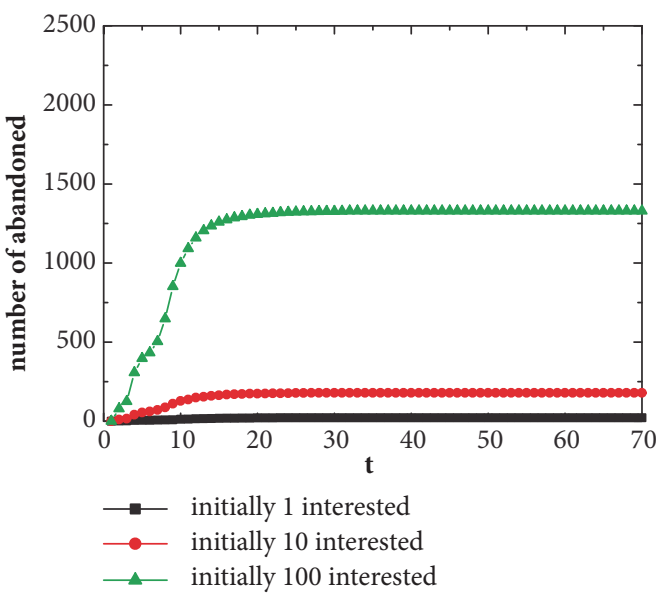

(a)

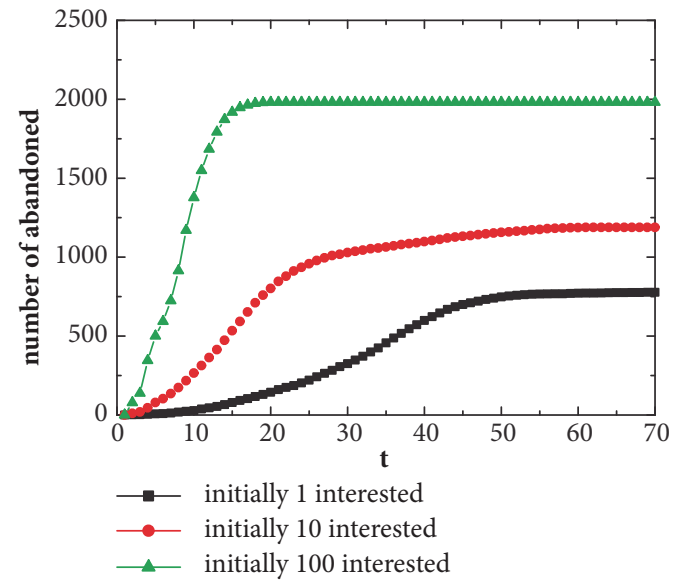

(b)

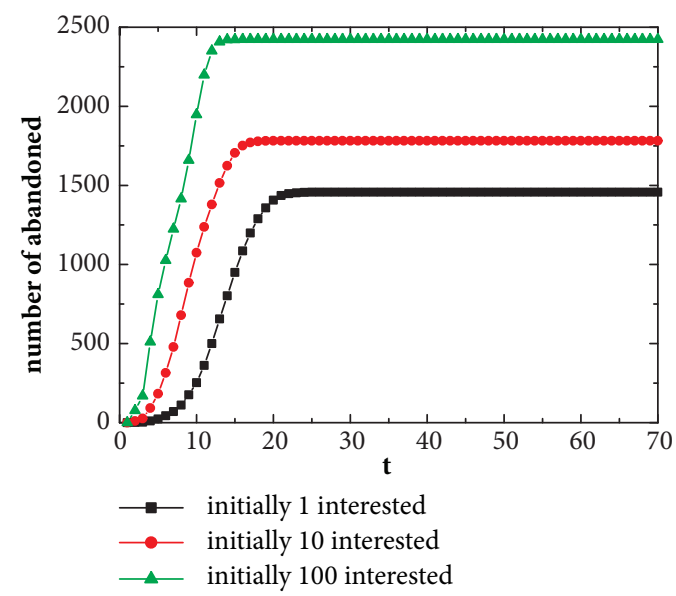

(c)

FIGURE 5: The influence of different initial infection ratio in small-word. The rewiring probability is $0,0.1,1$ in (a), (b), (c), respectively. Each experiment randomly selects 1, 10, 100 initial interested nodes.

contrast between Figures 4(b) and 4(d) shows that the abandoned nodes in SEIR are in the large area, while the number of immunized nodes in OSIS is flat. The results are consistent with the above conclusions.

4.2.2. The Influence of Different Initial Infection Ratio. In order to prove the influence of initial infection ratio on information spreading, we randomly select 1,10 , and 100 initial interested nodes. The rewiring probability in each set of experiments is 0, 0.1, and 1, respectively, and the infection rate is 0.35 . The threshold of the memory superposition factor is 1 and the threshold of information attenuation is 0.5 . The abandoned state nodes are recorded.

It is observable from Figure 5(a) that the number of initial infected nodes plays a decisive role in spreading when smallworld effect is not obvious (i.e., rewiring probability is 0 ). When the number of initial interested nodes is 10 , the number of the eventually abandoned nodes is 8 times more than that of 1 initial interested node, while the multiple is 60 times when the number of initial interested nodes is 100 and 1 . As shown in Figures 5(b) and 5(c), a higher rewiring probability leads to a faster spreading and a higher steady-state infection density. When the rewiring probability is 0.1 , the peak is reached at the 15 th time period, while it reduces to 10th time period when the rewiring probability is 1 . All the results above reveal that the more initial infected nodes, the easier it is to spread information. However, this effect tends to converge as the rewiring probability increases. Additionally, with the strengthening of the small-world features (i.e., the average number of friends per person in social networks is greater), the information can spread farther and inhibited harder.

4.2.3. The Effect of Memory Superposition. To explore the memory superposition effect on information spreading. We perform simulations to obtain the infection density in the steady state as a function of the effective infection rate $\alpha$ for various superposition thresholds. In this experiment, the rewiring probability of the network is 0.1 and the threshold of information attenuation is 0.5 .

We set up three sets of experiments. The infection rate in each set is $0.1,0.5$, and 0.7 ; meanwhile, the superposition threshold is 1,3 , and 5 . Initially, one node in network is 


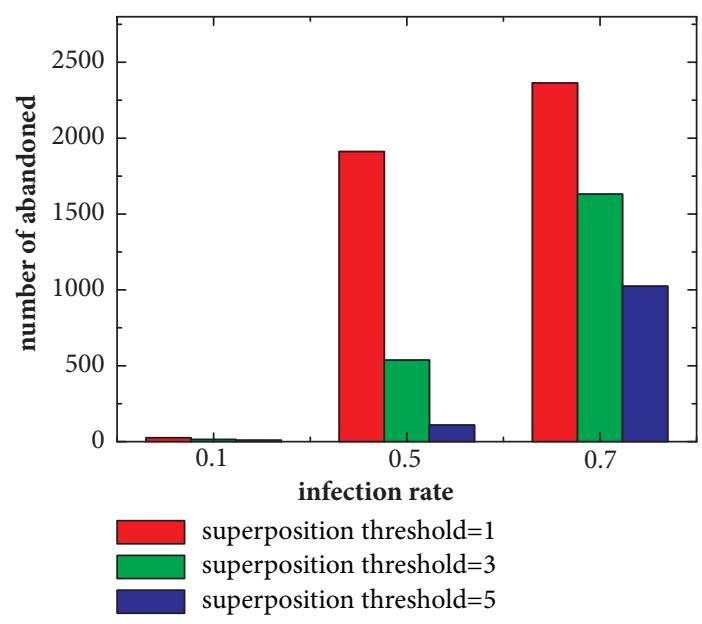

Figure 6: The number of abandoned node under different superposition threshold.

randomly chosen to be infected. After 100 time periods, the number of nodes in abandoned state is recorded.

As shown in Figure 6, the results of the experiments all indicate that the effect of superposition is obvious, and the higher the threshold, the fewer the nodes affected. For example, when the infection rate is 0.5 , the number of abandoned nodes with the threshold 1 is almost 4 times more than that with threshold 3, which even goes to 19 times when compared with the result of the threshold 5 . The results accurately explain why information spreads widely in online social networks. With the explosion of Internet and wemedia, the spreading of information costs little investigation and individuals spread information with less filters. Such less frequent contact to information leads to the rapid spreading. Memory superposition factor in our model stands for the number of contacts.

\section{Conclusions}

In summary, we introduce an OSIS model to describe information spreading in online social networks. Firstly, we propose OSIS by combining epidemics SEIR model with individual's cognitive psychology. Secondly, a cellular automata model of information transmission is designed, which provides a computational method for information spreading from a microscopic perspective. Additionally, we use smallworld network to simulate online social network, which verifies the spreading and evolution of information. The experimental results reveal that our model is consistent with the propagation of information in online social networks. We find that individual's cognition affects the information spreading behavior. When the infection rate is low, cognitive psychology prevents the spread of information. By contrast, when the infection rate is sufficiently high, it promotes transmission. We demonstrated that the explosion of online social network scale and the convenience of we-media can greatly increase the ability of information dissemination. Meanwhile, our research shows that the demise of information is affected by the dual effects of the decay of time and heat.

Our current work mainly concentrates on the spreading model itself. As for the future work, we believe that the real online social network is not a single small-world network. Due to the vigorous development of Web 2.0, online networks such as Weibo, Twitter, and Facebook are intertwined. We deem the information spreading in interconnected smallworld networks as the promising and challenging future work.

\section{Data Availability}

The data used to support the findings of this study are available from the corresponding author upon request.

\section{Conflicts of Interest}

The authors declare that they have no conflicts of interest.

\section{References}

[1] A. L. Barabási and R. E. Crandall, "Linked: the new science of networks," American Journal of Physics, vol. 71, no. 4, pp. 409411, 2003.

[2] M. E. J. Newman, "The structure and function of complex networks," SIAM Review, vol. 45, no. 2, pp. 167-256, 2003.

[3] A. Barrat, M. Barthlemy, and A. Vespignani, Dynamical processes on complex networks, 2008.

[4] R. Cohen and S. Havlin, "Complex networks: structure, robustness and function. London, UK," Cambridge University Press, 2010.

[5] P. Erdős and A. Rényi, "On the evolution of random graphs," Transactions of the American Mathematical Society, vol. 286, no. 1, pp. 257-274, 2011.

[6] D. J. Watts and S. H. Strogatz, Collective Dynamics of 'SmallWorld' Networks, 2011.

[7] A. Barabasi and R. Albert, "Emergence of scaling in random networks," Science, vol. 286, no. 5439, pp. 509-512, 1999.

[8] M. Li, Y. Fan, D. Wang, D. Li, J. Wu, and Z. Di, "Small-world effect induced by weight randomization on regular networks," Physics Letters A, vol. 364, p. 488, 2007.

[9] Q. Jin, L. Wang, C. Xia, and Z. Wang, "Spontaneous symmetry breaking in interdependent networked game," Scientic Reports, vol. 4, p. 4095, 2015.

[10] R. Pastor-Satorras and A. Vespignani, "Epidemic spreading in scale-free networks," Physical Review Letters, vol. 86, no. 14, pp. 3200-3203, 2001.

[11] N. Bailey, "The mathematical theory of infectious diseases and its applications," The Journal of Immunology, vol. 34, no. 5, pp. 955-956, 1978.

[12] R. M. Anderson and R. M. May, Infectious Diseases of Humans: Dynamics and Control, Oxford University Press, 1991.

[13] N. C. Grassly and C. Fraser, "Mathematical models of infectious disease transmission," Nature Reviews Microbiology, vol. 6, no. 6, pp. 477-487, 2008.

[14] R. M. May and A. L. Lloyd, "Infection dynamics on scale-free networks," Physical Review E: Statistical, Nonlinear, and Soft Matter Physics, vol. 64, no. 6, Article ID 066112, 2001. 
[15] M. H. A. Biswas, L. T. Paiva, and M. D. R. D. Pinho, "A SEIR model for control of infectious diseases with constraints," Mathematical Biosciences \& Engineering, vol. 11, no. 4, p. 761, 2014.

[16] X. Fu, A. Passarella, D. Quercia, A. Sala, and T. Strufe, "Online Social Networks," Computer Communications, vol. 73, pp. 163166, 2016.

[17] X. Ke, Z. Sai, C. Hao et al., "Measurement and analysis of online social networks," Chinese Journal of Computers, pp. 29-42, 2014.

[18] Q. Ying, Z. Nan, and Z. Laijun, "Research on the law of weibo publicity transmission," Journal of the China Society for Scientic and Technical Information, vol. 31, no. 12, pp. 1299-1304, 2012 (Chinese).

[19] weibo, https://weibo.com/.

[20] F. Xiong, Y. Liu, Z. J. Zhang, J. Zhu, and Y. Zhang, "An information diffusion model based on retweeting mechanism for online social media," Physics Letters A, vol. 376, p. 2103, 2012.

[21] D. Centola, "The spread of behavior in an online social network experiment," Science, vol. 329, no. 5996, pp. 1194-1197, 2010.

[22] $\mathrm{H}$. Wu, A. Arenas, and S. Gmez, "Influence of trust in the spreading of information," Physical Review, vol. 95, 2016.

[23] A. L. Hill, D. G. Rand, M. A. Nowak, and N. A. Christakis, "Infectious disease modeling of social contagion in networks," PLoS Computational Biology, vol. 6, no. 11, Article ID e1000968, 15 pages, 2010.

[24] L. Lü, D.-B. Chen, and T. Zhou, "Small world yields the most effective information spreading," New Journal of Physics, p. 825834, 2011.

[25] J. X. Zhang, D. B. Chen, Q. Dong, and Z. D. Zhao, "Identifying a set of influential spreaders in complex networks," Scientic Reports, vol. 6, 2016.

[26] W. Wang, M. Cai, and M. Zheng, "Social contagions on correlated multiplex networks," Physica A Statistical Mechanics \& Its Applications, vol. 499, 2017.

[27] W. Wang, X. L. Chen, and L. F. Zhong, "Social contagions with heterogeneous credibility," Physica A Statistical Mechanics \& Its Applications, 2018.

[28] W. Wang, M. Tang, S. H. Eugene, and L. A. Braunstein, "Unification of theoretical approaches for epidemic spreading on complex networks," Reports on Progress in Physics, p. 036603, 2017.

[29] W. Wang, M. Tang, H. F. Zhang, H. Gao, Y. Do, and Z. H. Liu, "Epidemic spreading on complex networks with general degree and weight distributions," Physical Review E Statistical Nonlinear \&a Soft Matter Physics, vol. 90, p. 042803, 2014.

[30] X. Zhu, W. Wang, S. Cai, and H. E. Stanley, "Stanley, Dynamics of social contagions with local trend imitation," Scientic Reports, vol. 8, 2018, Scientic Reports.

[31] X. Zhu, W. Wang, S. Cai, and H. E. Stanley, "Optimal imitation capacity and crossover phenomenon in the dynamics of social contagions," Journal of Statistical Mechanics Theory \& Experiment, Article ID 063405, p. 063405, 2018.

[32] A. L. Barabsi, R. Albert, and H. Jeong, "Mean-field theory for scale-free random networks," Physica A, vol. 272, p. 173, 1999.

[33] S. A. Pandit and R. E. Amritkar, "Characterization and control of small-world networks," Physical Review E: Statistical, Nonlinear, and Soft Matter Physics, vol. 60, no. 2, pp. R1119-R1122, 1999.

[34] J. Von Neumann, Papers of John Von Neumann on Computing \& Computer Theory, vol. 1, 1951.

[35] B. Chopard and M. Droz, Cellular Automata for Modeling Physics, Cambridge University Press, Cambridge, UK, 1998.
[36] G. J. Martnez, A. Adamatzky, F. Chen, and L. Chua, "On Soliton Collisions between Localizations in Complex Elementary Cellular Automata: Rules 54 and 110 and Beyond," Complex Systems, vol. 117, 2013.

[37] H. F. Gagliardi and D. Alves, "Small-world effect in epidemics using cellular automata," Mathematical Population Studies. An International Journal of Mathematical Demography, vol. 17, no. 2, pp. 79-90, 2010.

[38] X. Jin, J. Li, and L. Zhang, Online Social Networks Based on Complex Network Theory and Simulation Analysis, Springer International Publishing, 2015. 


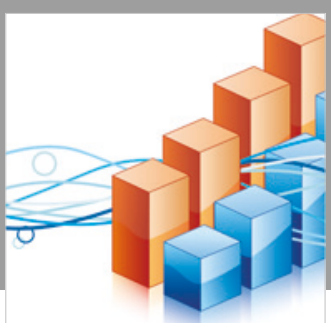

Advances in

Operations Research

\section{-n-m}
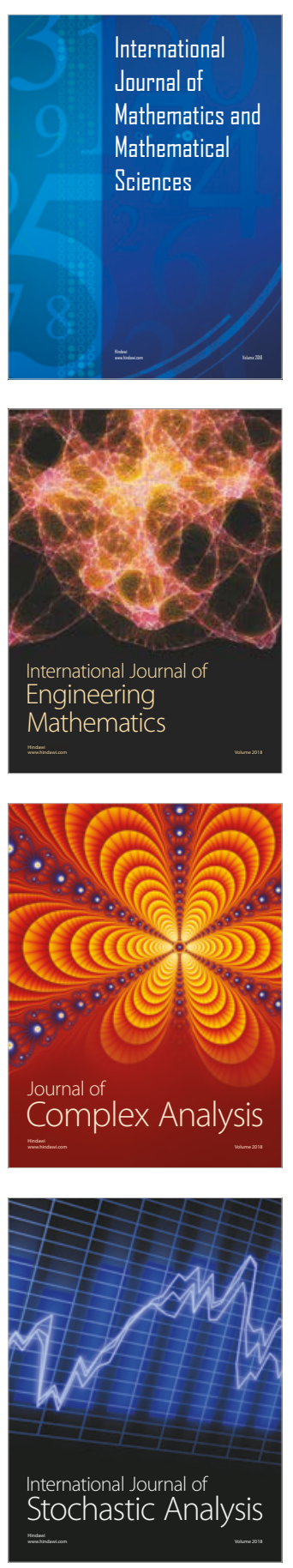
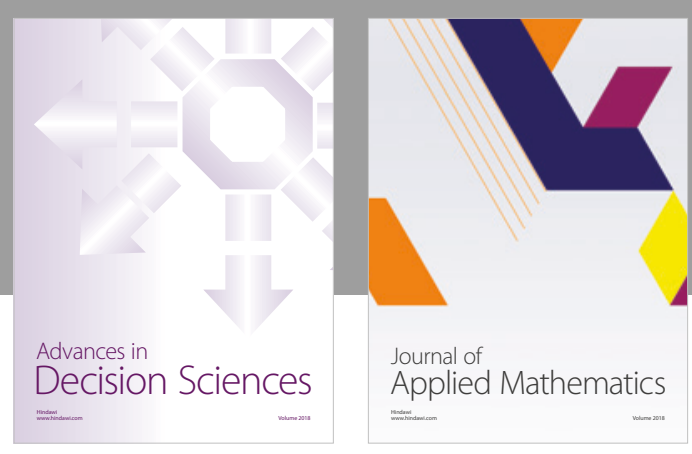

Journal of

Applied Mathematics
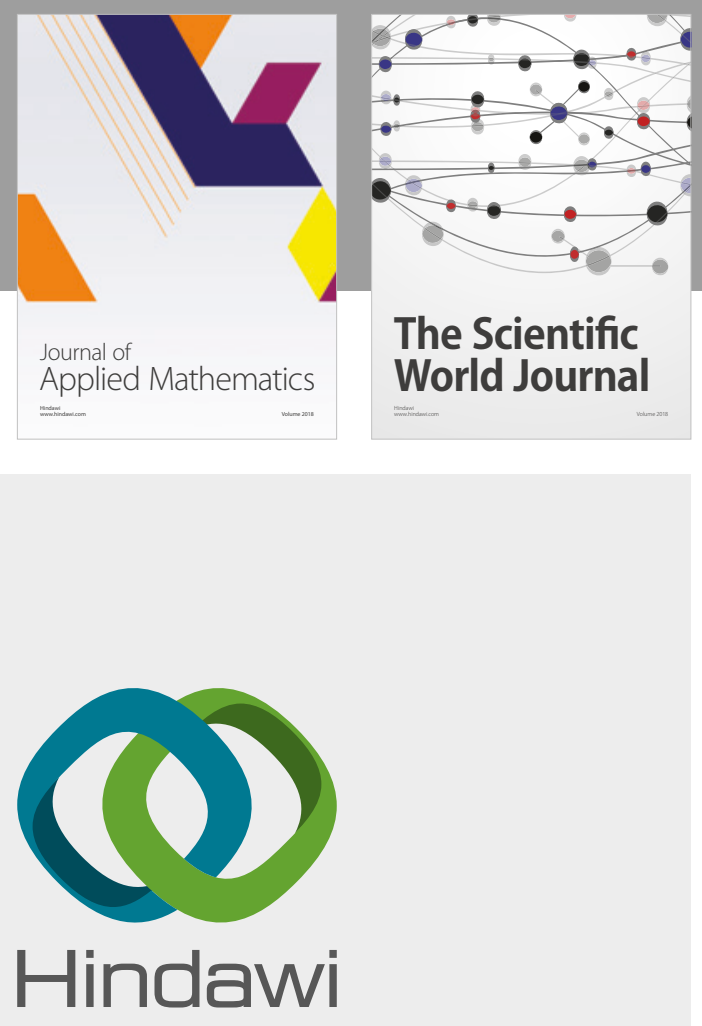

Submit your manuscripts at

www.hindawi.com

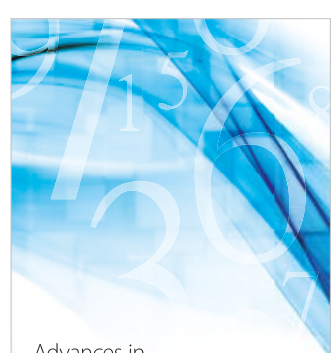

Advances in
Numerical Analysis
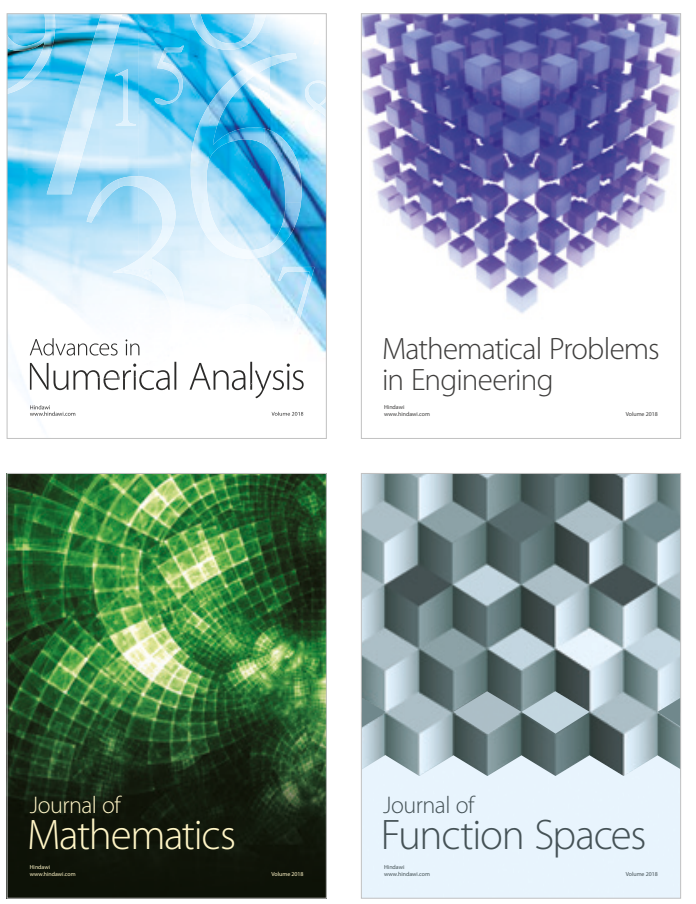

Mathematical Problems in Engineering

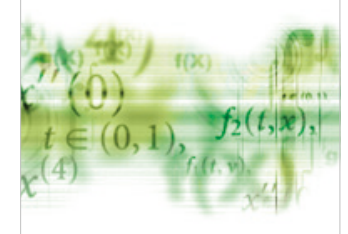

International Journal of

Differential Equations

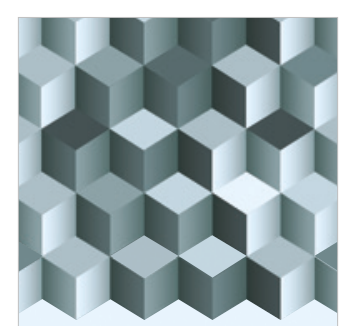

Journal of

Function Spaces
The Scientific

World Journal

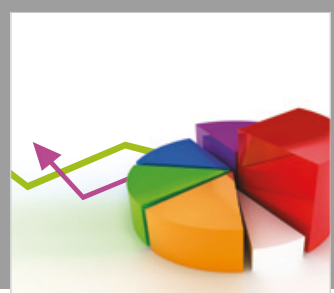

Journal of

Probability and Statistics
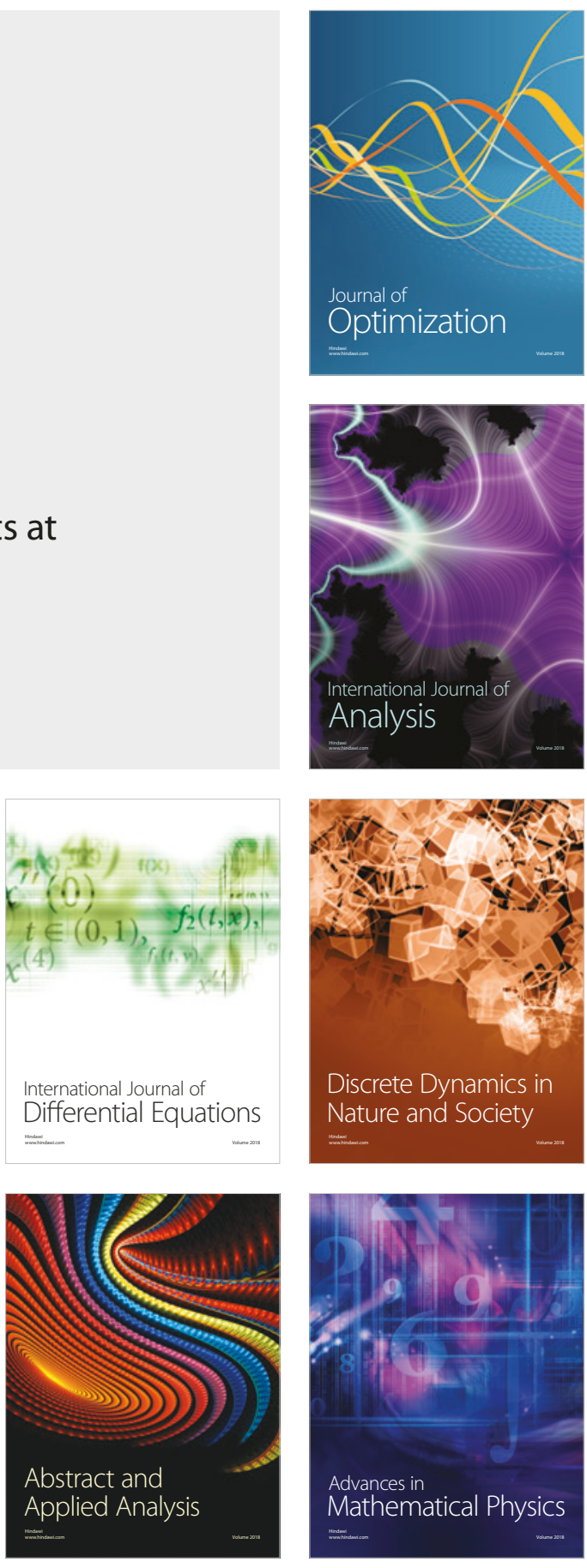